\title{
Rosselli-Gulienetti Syndrome: Clinical Presentation and Management - A Rare Case Report
}

\author{
Vedantham Madhusudhana Rao, Basapogu Sreeramulu, Dasari Mounika, \\ Koppula Prathyusha, Mansha Saxena \\ Department of Prosthodontics, Government Dental College and Hospital, Hyderabad, Telangana, India
}

Email for correspondence: madhu.vedantham@gmail.com

\begin{abstract}
Rosselli-Gulienetti syndrome (RGS) is an inherited genetic disease, follows an autosomal recessive pattern of inheritance. This is a case of completely edentulous RGS with incomplete closure of the roof of the mouth and a cleft lip with an oronasal communication. The presence of an oronasal fistula presents a challenge to maxillary complete denture fabrication because leakage of air from the nasal cavity through the fistula prevents the formation of an adequate border seal and further absence of an anterior portion of alveolar ridge and lip, worsen the retention of the denture. The overall goal of rehabilitating such patients is focused on the closure of oronasal communication, improving acceptable speech, appearance, proper occlusion, and masticatory function. The present clinical report illustrates an alternative solution by integrating a small retentive component into a maxillary complete denture.
\end{abstract}

Key words: Oronasal fistula, Rosselli-Gulienetti syndrome, silicone material, stainless steel retainers

\section{INTRODUCTION}

Rosselli-Gulienetti syndrome (RGS) is an extremely rare congenital disorder and its exact incidence is unknown. It was first described by the researchers Rosselli and Gulienetti in 1961. ${ }^{[1]}$ Males and females are affected in equal numbers. Its incidence tends to be higher in children of parents who are consanguineous (closely related or sharing genetic material).

RGS is a form of ectodermal dysplasia and its symptoms include reduced or absent ability to sweat, facial abnormalities and defects in the hair, teeth, nails, fingers, toes, and urinary tract. Specifically, symptoms of RGS include cleft lip or palate, intellectual disability, fused eyelids,

\begin{tabular}{l} 
Quick Response Code Article Info: \\
\hline $\begin{array}{l}\text { Roi: 10.5866/2018.10.10201 } \\
\text { Received: } 24-09-2018 \\
\text { Revised: } 27-10-2018 \\
\text { Accepted: } 23-11-2018 \\
\text { Available Online: 05-01-2019, (www. } \\
\text { nacd.in)@ NAD, } 2019 \text { - All rights reserved }\end{array}$ \\
\hline
\end{tabular}

absent nails, delayed bone growth, urogenital abnormalities, and dry skin. ${ }^{[2]}$

There are reports that it is caused by a mutation or defect in the PVRL-1 gene, which provides instructions for making the protein nectin-1. Nectin-1 is an immunoglobulin-like adhesion molecule that is widely expressed in epithelial tissues and it participates in forming the adherens junctions, which serve to connect cells within the skin. $^{[3]}$

The long-term prognosis for people with RGS is largely unknown because there are so few cases. If symptoms are managed and complications prevented, people with RGS should enjoy long, healthy lives. ${ }^{[3]}$

An oronasal fistula is an epithelialized communication between the oral and the nasal cavity. This defect may effect speech and cause nasal regurgitation. The presence of this defect complicates the provision of a prosthesis for edentulous patients because no natural teeth are present to provide retention; as retention depends primarily on atmospheric pressure combined with 
intimate tissue contact and a peripheral border seal. ${ }^{[4]}$

In the present case report, the patient presented with a palatal defect, despite previous attempts of surgical repair, with an oronasal fistula. Even a technically perfect maxillary denture cannot achieve a suction effect as a result of breakage of the seal from air passing through the fistula. Hence, an alternative prosthetic solution was reached by integrating a small retentive component into the oronasal fistula of the maxillary complete denture with adequate retention for the patient. ${ }^{[5]}$

\section{CASE REPOR}

A 23-years-old male patient was referred to the department of prosthodontics, for the rehabilitation of completely edentulous maxillary and mandibular arches with oronasal communication. On eliciting history, the patient had undergone reconstructive cleft lip and palate surgery twice, when he was a child and later when he was 15-years-old. "V" shaped oronasal fistula was remained at the midline near the incisive canal, in spite of surgical attempts to close the oronasal communication. Stricture formation at the surgical site has obliterated the labial sulcus and restricted the upper lip movement in the anterior region. The patient had recently undergone extraction of remaining poor prognosis teeth in the upper and lower arches. On clinical examination, both maxillary and mandibular edentulous arches were found to be severely resorbed with loss of alveolar bone in the maxillary anterior region [Figure 1]. The posterior part of the hard palate, where the graft was placed during surgery is observed to be very irregular, soft, and redundant.

To record the undercut, preliminary impression of the maxilla was made using irreversible hydrocolloid impression material (Algitex, DPI, Mumbai) and for mandible, impression compound (Y-DENTS impression composition, MDM corporation, Delhi) was used. Special trays were fabricated for border molding by giving clearance in the maxillary anterior region. After peripheral tracing with green stick compound (DPI PINNACLE tracing sticks, Mumbai), definitive impressions were made with addition silicone light body impression material (Photosil, DPI, Mumbai) to capture the details of soft tissue undercuts accurately within the fistula. Master casts were made using dental stone (Neelkanth STONE PLASTER, Jodhpur, India) [Figure 2].
Conventional steps in complete denture fabrication were followed until recording of jaw relations and transferred to an articulator. The relief was given in the anterior part of the maxillary occlusal rim (No 2, Y-DENTS modeling wax, Delhi) to accommodate surgical defect so that it does not interfere with the stability of the prosthesis [Figure 3]. Try in was done and occlusion was evaluated [Figure 4].

One loop-shaped and one C-shaped, $0.8 \mathrm{~mm}$ stainless steel (Konark, Everbright Dental, India) retainers were customized to fit into the "V" shaped

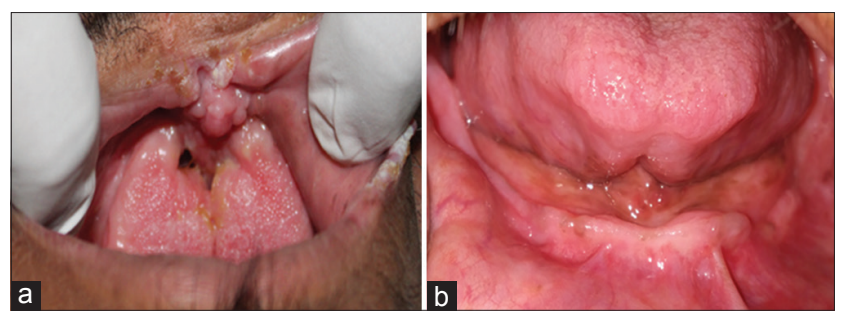

Figure 1: Clinical picture showing both maxillary (a) and mandibular (b) edentulous arches which were found to be severely resorbed with loss of alveolar bone and fistula in the maxillary anterior region

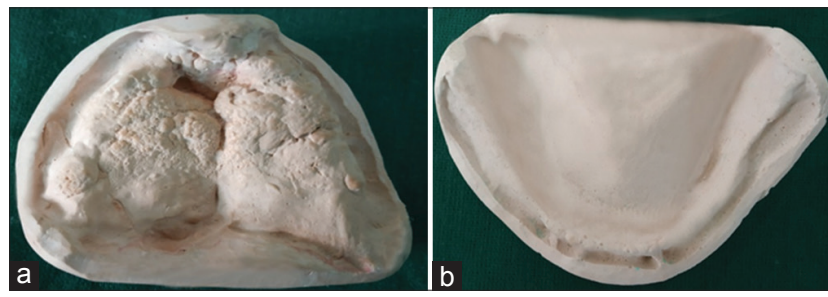

Figure 2: Maxilla (a) and mandible (b) master casts were prepared using dental stone

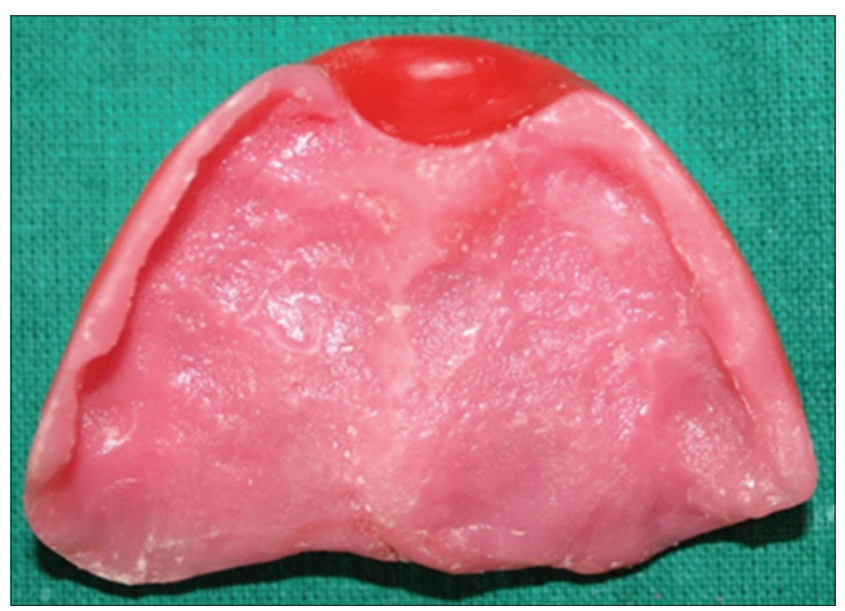

Figure 3: Relief was given in the occlusal rim to accommodate anterior defect (stricture) 
oronasal defect of the master cast, which will provide support for silicone relining material [Figure 5].

Prosthetic treatment was planned by elaborating a removable acrylic prosthesis best suited for the patient, with a palatal obturator of permanent soft silicon based relining material to improve denture retention and stability. Retainers were used to support the relining material and reproduce the palatal defect. The purpose of the wire loops was to retain the obturator into the prosthesis. The dentures were acrylized using heat cure veined

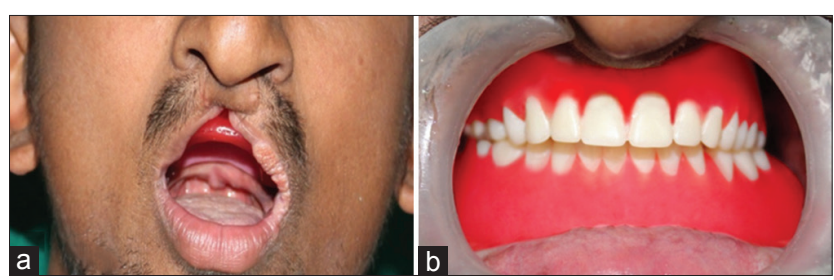

Figure 4: Recording of jaw relations (a) and try in was done (b).

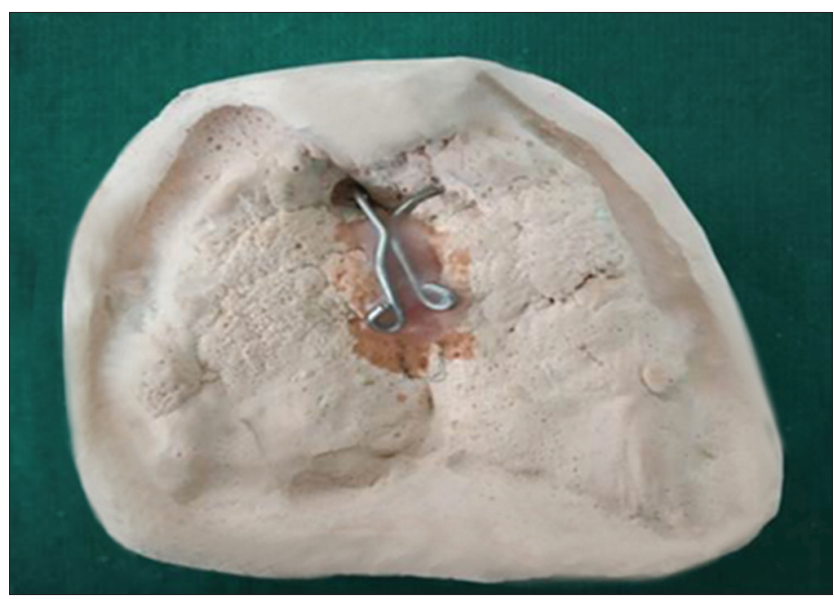

Figure 5: Stainless steel retainers in position

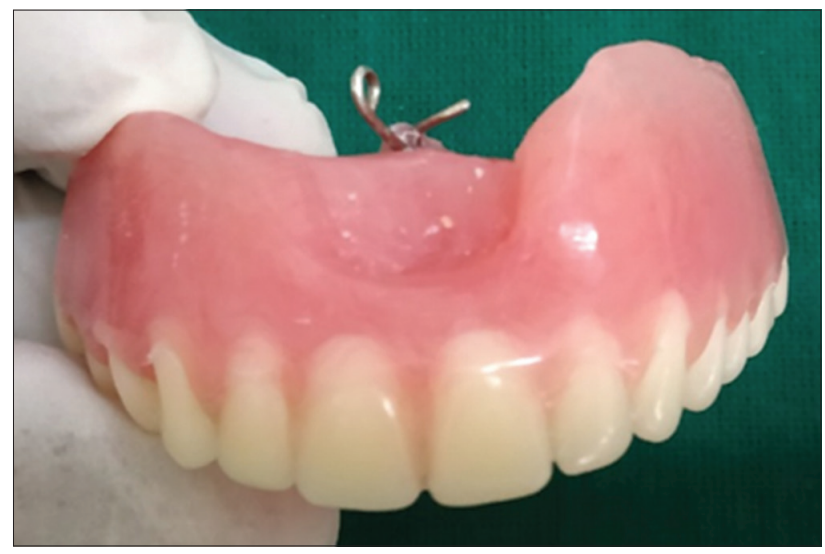

Figure 6: Retainers incorporated in denture acrylic resin (ACRYLYN-H denture material, Mumbai) using routine procedures by incorporating the retainers, then finished and polished [Figure 6]. The fit of the dentures was checked clinically and radiologically (lateral cephalogram) by placing the dentures in the patient mouth and evaluated.

The maxillary denture was relined in the oronasal fistula area with permanent soft siliconebased relining material (GC RELINE Soft), with the chairside relining procedure [Figure 7] This material was retained by the stainless steel retainers, which entered the palatal hole and increased the retention of the denture. The material was withdrawn from the mouth before it had fully set to cutoff excess material and shape it into a retentive but not harmful design. To avoid dehiscence of the antrum, we checked that harmful pressure forces were properly alleviated and

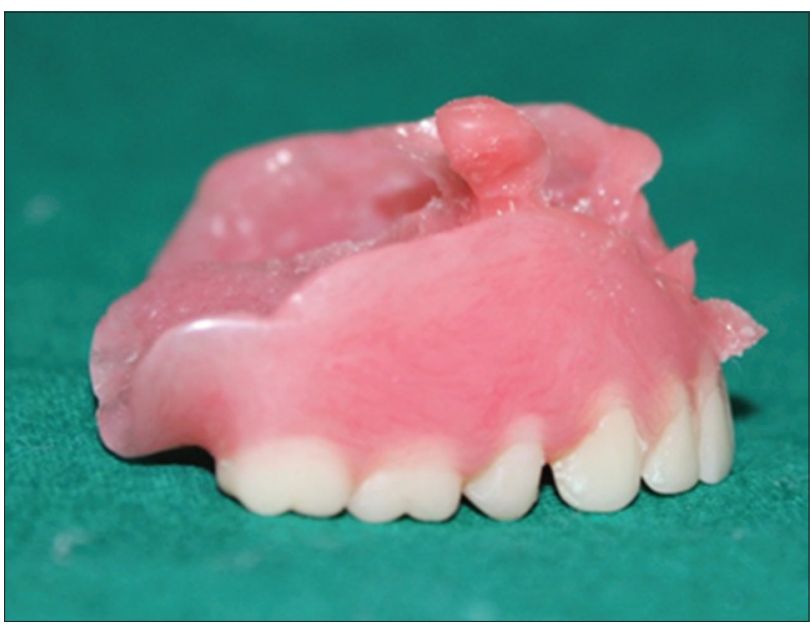

Figure 7: Relining of the maxillary denture in the oronasal fistula area with permanent soft silicone-based relining material

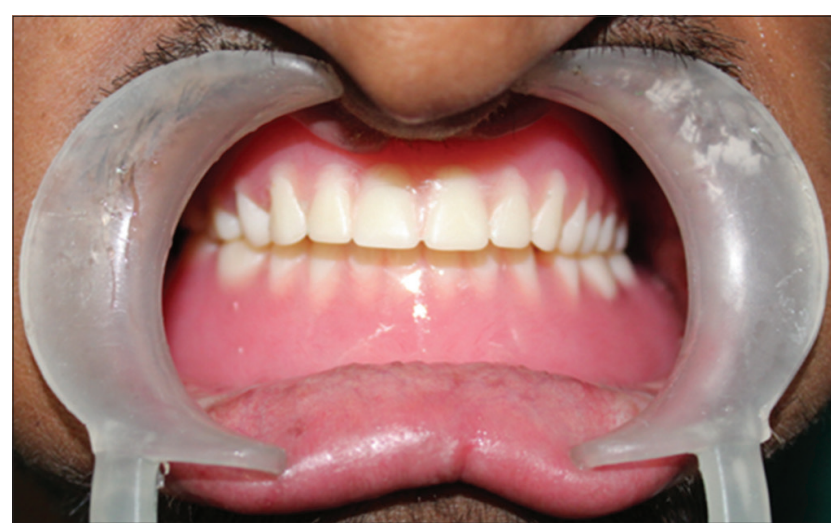

Figure 8: Insertion of the prosthesis in the patient's mouth 
relieved from the communication. It can be checked visually by examination of soft tissue blanching and extension of the wire components into the defect by lateral cephalogram.

After finishing and polishing, the prosthesis was inserted in the patient's mouth and was seen to provide enough retention and stability to provide optimal chewing capacity and comfort [Figure 8]. Prosthesis obtained retention from defect area undercut that there is no interference with the path of insertion and removal of appliances and does not cause any pressure or pain on the soft tissue of the patient. There was also a remarkable improvement in speech and esthetics. Both the dentures were delivered to the patient. The patient was educated in the care and maintenance of the prosthesis. Long-term regular follow-up appointments were carried out since soft silicone relining material hardens over a period of time and requires replacement.

\section{DISCUSSION}

RGS was first described by Rosselli and Gulienetti in 1961. The exact etiopathogenesis is unknown. Most of the authors believe that RGS is due to a genetic disorder (Due to mutation or defect in the PVRL-1 gene which provides instructions for making the protein nectin-1). ${ }^{[1]}$ The symptoms, in this case, were reduced ability to sweat, scanty hair, missing teeth, cleft lip and palate, fused eyelids and dry skin, fissuring of the feet, scales on dorsum of the feet, and diffuse hyperkeratotic hyperpigmented plaques over soles.

The differential diagnosis of this syndrome includes ectodermal dysplasia and Papillon-Lefevre syndrome. The features of ectodermal dysplasia are hypohydrosis, hypotrichosis, and hypodontia, whereas in Papillon-Lefevre syndrome hypodontia with periodontopathy is seen. ${ }^{[6]}$

The treatment planning of this syndrome should include a team of dermatologists and dental surgeons. ${ }^{[7]}$ Vitamins A and D, Moisturex and Levocetirizine tablets are used to treat both the cutaneous and the dental defects. The prosthetic approach is an age specific treatment involving the fabrication of partial or complete dentures initially. At an early age, during mixed dentition period, missing teeth are replaced with a removable prosthesis and regular follow-up of the patient has to be carried out for maintaining esthetics and functioning. ${ }^{[8]}$ The prosthodontic management strategy for young edentulous patients in general, must include the preservation of the alveolar ridge, keeping the vertical dimension of the occlusion low and the use of narrow posterior teeth.

Review of literature suggests several treatment modalities such as conventional complete dentures, modified complete dentures, implant-supported complete dentures, overdentures or a combination of these. Treatment of medically compromised completely edentulous cleft lip and cleft palate patient with oronasal communication provides a tremendous challenge to the prosthodontists, as the prosthesis shows a lack of retention due to their weight and the inability to achieve a proper sealing of the defect. The type of material to be used to create obturator prosthesis depends on several factors, such as the type and extent of the fistula and the ability of the patient to cope with conventional removable dentures, although the aim was always to restore the anatomical loss of hard and soft tissues, providing enough retention to the denture to improve functional and psychological well-being. ${ }^{[9]}$

Prosthetic rehabilitation with a palatal obturator is mainly indicated when the closure of the fissure is not complete after corrective surgery. It seems that changes related to esthetics, function and psychological well-being has an impact on a patient's personal lives and this is also a great satisfaction for caregivers. This clinical report describes an alternate non-invasive prosthetic treatment for an edentulous patient with an oronasal communication where the patient declines surgery or where surgical procedures are otherwise contraindicated. Prosthetic rehabilitation with an obturator prosthesis is thus a conservative, economical and quicker method and has lesser clinical complications than an implant-retained prosthesis. $^{[10]}$

\section{CONCLUSION}

RGS is a rare genetic disorder. It is important that dental professionals be familiar with the RGS-specific dermatologic manifestations, to be able to identify the disease in its very early stages. If symptoms are managed and complications prevented, people with RGS can lead a long and healthy life. This should prevent or delay tooth loss and enhances early replacement of missing teeth for the preservation of function and esthetics. This case report suggests an alternative solution 
by integrating a small retentive component into a maxillary complete denture, in a cleft lip and cleft palate patient with an oronasal communication to increase its retention and stability.

\section{Declaration of patient consent}

The authors certify that they have obtained appropriate patient consent for the use of images and other clinical information. Patient understands that due efforts have been made to conceal their identity, but anonymity cannot be guaranteed.

\section{REFERENCES}

1. Rosselli D, Gulienetti R. Ectodermal dysplasia. Br J Plast Surg 1961;14:190-204.

2. Ladda RL, Zonana J, Ramer JC, Mascari MJ, Rogan PK Congenital contractures, ectodermal dysplasia, cleft lip/palate, and developmental impairment: A distinct syndrome. Am J Med Genet 1993;47:550-5.

3. Online Mendelian Inheritance in Man. Rosselli Gulienetti Syndrome and Bowen Armstrong Syndrome. Baltimore: Online Mendelian Inheritance in Man (OMIM); 2006.
4. Law MY, Chung RW, Lam OL. Prosthetic rehabilitation of an edentulous patient with an oronasal fistula. J Prosthet Dent 2015;113:347-9

5. Montero J, Macedo C, Rodriguez M, López-Valverde A. Prosthetic rehabilitation of an edentulous cleft palate using a denture with a palatal obturator: A clinical report. J Clin Exp Dent 2011;3:365-8.

6. Jain V, Gupta R, Prakash H. Prosthodontic rehabilitation in papillon lefevre syndrome: A case report. J Indian Soc Pedod Prev Dent 2005;23:96-8.

7. Sreeramulu B, Haragopal S, Shalini K, Sudha MD, Kiran G The prosthodontic management of a young edentulous patient with the papillon lefevre syndrome-a rare case report. J Clin Diagn Res 2012;6:1808-11.

8. Sreeramulu B, Shyam ND, Ajay P, Suman P. Papillonlefèvre syndrome: Clinical presentation and management options. Clin Cosmet Investig Dent 2015;7:75-81.

9. Ismail IJ, Jani GH. Maxillofacial prosthestic treatment of congenital craniofacial defect patients. Int J Adv Res 2016;4:937-41.

10. Ferreira AN, Prithika E, Aras M, Chitre V, Coutinho I. Prosthodontic rehabilitation of a completely edentulous cleft palate patient using an unconventional complete denture a case report. Dentistry 2018;8:483. 